\title{
Cognitive behavioural therapy improves psychotic symptoms at 18 months in people with schizophrenia
}

Tarrier N, Lewis S, Haddock G, et al. Cognitive-behavioural therapy in first-episode and early schizophrenia. 18-month follow-up of a randomised controlled trial. Br J Psychiatry 2004; 184:231-9.

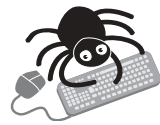

This article

contains extra text on the EBMH website

Does cognitive behavioural therapy improve symptoms in people with schizophrenia?

METHODS

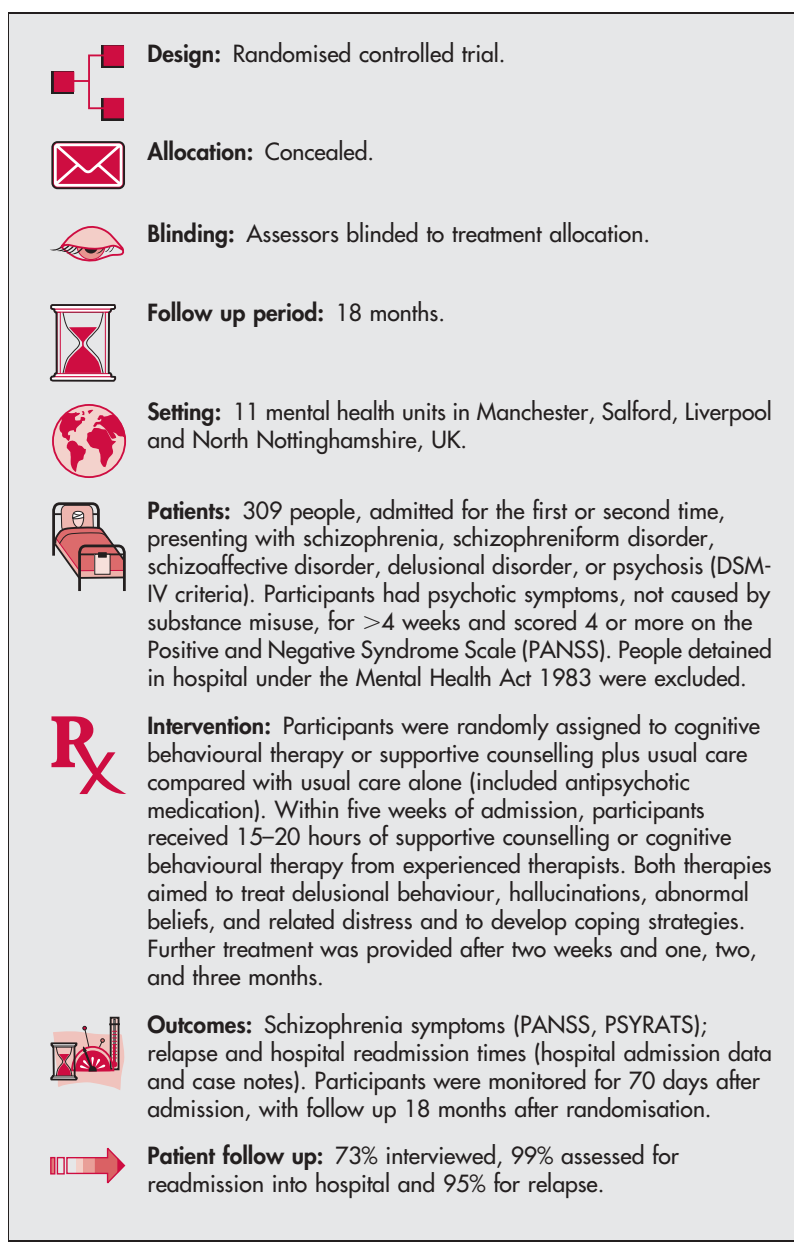

\section{MAIN RESULTS}

At 18 months both cognitive behavioural therapy and supportive counselling plus usual care significantly improved schizophrenia symptoms compared with usual care $(\mathrm{p}>0.005)$. There were no significant differences in hospital readmission or relapse rates among groups (see http://www.ebmentalhealth.com/supplemental for table). There was no significant difference between cognitive behavioural therapy and supportive counselling in symptom improvement $(\mathrm{p}>0.725)$. There were no significant differences in medication use, type, and dosage among groups.

For correspondence: Nicholas Tarrier, Education and Research Building, Wythenshawe Hospital, Manchester, UK; nicholas.tarrier@man.ac.uk

Sources of funding: the UK Medical Research Council, Northwest England Regional NHSE Office, Trent Regional NHSE Office and Manchester, Salford and Trafford, Liverpool, Sefton, St Helens and Knowsley and North Nottinghamshire Health Authorities.

\section{CONCLUSIONS}

Cognitive behavioural therapy and supportive counselling significantly improve schizophrenia symptoms compared with usual care. However, neither of the therapies affected relapse or rehospitalisation rates in the long term.

\section{Commentary}

D espite cognitive-behavioural therapy (CBT) being increasingly used to treat psychotic symptoms in medication resistant clients and chronic schizophrenia, use in first episode or "critical stages" has not been extensively researched.' The Tarrier et al paper provides a welcome and valuable contribution to the literature on early intervention.

Although there was lack of differentiation between CBT and supportive counselling at follow up, both provided significant symptom reduction over usual treatment. The lack of difference in relapse or hospitalisation rates across the two treatments and control group was unexpected. Previous research shows an association between duration of untreated psychosis and outcome, ${ }^{2}$ and as this study focuses on a "critical" period, the ultimate course of the disorder may have been altered. This would potentially be reflected outside of this study's 18 month follow up. The "concealment" of group allocation from both assessors and therapists maintained appropriate control groups and improved on comparison. Previous studies have not always had this methodological rigour, with resultant opaqueness concerning intervention and control group contrast. Long term, 18 month follow up is an important indicator of the "maintenance of change" associated with treatment in this study. A longer term examination of symptom change, relapse, and rehospitalisation rates as a result of CBT (and the surprisingly robust supportive counselling condition) would be beneficial. That said, this study highlights the importance of deploying psychological therapy during the phase of the illness in which it is most likely to be of benefit in minimising the possibly devastating, even fatal, effects of the disorder.

As treatment priorities vary over the course of the illness, a flexible integration of biological, social, and psychological interventions is necessary. ${ }^{3}$ As the supportive counselling condition was as effective at follow up as CBT, and was seen as "unstructured", this may highlight the need for not allocating $100 \%$ of contact time to "pure" therapy, thereby sustaining engagement and promoting associated clinical gain. Importantly, the multicentre approach in this study begins to reflect the "real world" application of treatment in varying contexts. An increased number of booster sessions spread over contact time, and progressively implemented in a community setting, would be advantageous. This may improve on long term adaptation of the intervention and generalisation, with potential reduction in relapse rates.

Although primary prevention of schizophrenia and related disorders is at present out of reach, this article reinforces the importance of early intervention. Early focus on impairment of functioning is an essential clinical task. To do otherwise weakens subsequent change and reduces potential functional gain for the individual.

Patrick K Kingsep, BA (Hons), MA (Clin Psych)

Centre for Clinical Interventions, Perth, Western Australia, Australia

1 Garety PA, Fowler D, Kuipers E. Cognitive-behavioural therapy for people with psychosis. In: Martindale D, Bateman A, Crowe M, et al, eds. Psychosis: psychological approaches and their effectiveness. London: Gaskell; 2000:30-49.

2 Norman RMG, Malla AK. Duration of untreated psychosis: a critical examination of the concepts and its importance. Psychol Med 2001;31:381-400

3 Edwards J, McGorry PD. Implementing early intervention in psychosis. London: Martin Dunitz Ltd, 2002. 